\title{
ALGEBRAIC RELATIONS AMONG SIX ADJACENT $\tau$-FUNCTIONS RELATED TO THE FOURTH PAINLEVÉ SYSTEM
}

\author{
Kazuo OKAMOTO \\ (Received 11 December 1995, and revised 14 March 1996)
}

\section{Introduction}

This paper considers the $\tau$-functions related to the Hamiltonian system:

$$
\frac{d q}{d t}=\frac{\partial H}{\partial p}, \quad \frac{d p}{d t}=-\frac{\partial H}{\partial q},
$$

where $H=H(t ; q, p)$ is given by the polynomial

$$
2 q p^{2}-\left(q^{2}+2 t q+2\left(v_{2}-v_{1}\right)\right) p+\left(v_{3}-v_{1}\right) q .
$$

Here $\boldsymbol{v}=\left(v_{1}, v_{2}, v_{3}\right) \in \mathbb{C}^{3}$ is a constant vector of parameters such that

$$
v_{1}+v_{2}+v_{3}=0
$$

For a solution $(q(t), p(t))$ of $(0.1)$, the $\tau$-function $\tau=\tau(t)$ related to the system is defined by

$$
\frac{d}{d t} \log \tau(t)=H(t ; q(t), p(t)),
$$

with ambiguity of a multiplicative constant. The Hamiltonian system (0.1)-(0.2) is called the fourth Painleve system, or simply, in this paper, the Painlevé system, since by eliminating $p$ from the system we obtain the fourth Painlevé equation $\mathrm{P}_{\mathrm{IV}}$ :

$$
\frac{d^{2} q}{d t^{2}}=\frac{1}{2 q}\left(\frac{d q}{d t}\right)^{2}+\frac{3}{2} q^{3}+4 t q^{2}+2\left(t^{2}-\alpha\right) q+\frac{\beta}{q},
$$

where $\alpha=3 v_{3}+1, \beta=-2\left(v_{2}-v_{1}\right)^{2}$, see [3]. The Hamiltonian structure associated to $(0.1)-(0.2)$ is the quartet

$$
\mathcal{H}=(q, p, H, t),
$$


which is also called the Painlevé system. When considering the Painlevé system at an arbitrarily fixed value $\boldsymbol{v}$ of the parameters, we write $(0.4)$ as

$$
\mathcal{H}(\boldsymbol{v})=(q(\boldsymbol{v}), p(\boldsymbol{v}), H(\boldsymbol{v}), t) .
$$

A solution $(q(v), p(v))=(q(t ; v), p(t ; v))$ of $(0.1)$ will be called that of $\mathcal{H}(\boldsymbol{v})$ for simplicity of presentation. Using such a solution, the $\tau$-function $\tau(v)=\tau(t ; v)$ related to $\mathcal{H}(v)$ is defined by $(0.3)$. It is known by [2] that $\tau(v)$ is an entire function on the whole complex plain $\mathbb{C}$ and that the $\tau$-function admits only simple zeros, that is,

$$
\text { if } \quad \tau\left(t_{0} ; v\right)=0, \quad \text { then } \frac{d \tau}{d t}\left(t_{0} ; v\right) \neq 0 .
$$

To describe the main results of this paper, we introduce some results obtained in [3]. First, in (0.1)-(0.2) put

$$
\begin{gathered}
q=-2 P \frac{P Q-v_{2}+v_{1}}{P Q-v_{3}+v_{1}+1}, \\
p-\frac{1}{2} q-t=\frac{P Q-v_{3}+v_{1}+1}{2 P},
\end{gathered}
$$

and define a function $K$ by $K=H+q$. Then we obtain

$$
K=2 Q P^{2}-\left(Q^{2}+2 t Q+2\left(v_{2}-v_{1}\right)\right) P+\left(v_{3}-v_{1}+1\right) Q .
$$

Moreover, the transformation from $(q, p, H, t)$ to $(Q, P, K, t)$ is birational and canonical, so that we have the birational canonical transformation

$$
\rho: \mathcal{H}(\boldsymbol{v})=(q, p, H, t) \longrightarrow \mathcal{H}(\ell(v))=(Q, P, K, t),
$$

where $\ell$ is the translation on the parameter space

$$
\ell(v)=v+e, \quad \text { with } \quad e=\frac{1}{3}(-1,-1,2) .
$$

By applying the transformation $\rho$ successively, we obtain the sequence $\left\{\mathcal{H}_{m} ; m \in \mathbb{Z}\right\}$ of the Hamiltonian structures such that

$$
\begin{aligned}
& \mathcal{H}_{0}=\mathcal{H}(\boldsymbol{v}), \\
& \mathcal{H}_{m}=\rho^{m} \mathcal{H}_{0}=\mathcal{H}\left(\boldsymbol{v}_{m}\right),
\end{aligned}
$$

where $\boldsymbol{v}_{m}=\ell^{m}(\boldsymbol{v})=\boldsymbol{v}+m \boldsymbol{e}$. Then the sequence $\left\{\tau_{m} ; m \in \mathbb{Z}\right\}$ of the $\tau$-functions is given by

$$
\frac{d}{d t} \log \tau_{m}=H_{m}
$$


It is known that the sequence of $\tau$-functions satisfies the Toda equation:

$$
\frac{d^{2}}{d t^{2}} \log \tau_{m}+2\left(v_{3}-v_{1}+m\right)=c(m) \frac{\tau_{m-1} \tau_{m+1}}{\tau_{m}^{2}}, \quad m \in \mathbb{Z},
$$

$c(m)$ being a nonzero constant. Since the $\tau$-function is determined up to a multiplicative constant, the $\tau$-functions, $\tau_{m}$, in $(0.5)$ can be replaced by $f(m) \tau_{m}$, $f(m)$ being nonzero constants. By selecting a suitable normalization of constants, we put $c(m)=1$ for all $m \in \mathbb{Z}$ without loosing generality.

Let $R=\mathbb{Z}[S, V]$ be the polynomial ring of the nine variables

$$
\boldsymbol{S}=\left(S_{0}, S_{1}, S_{2}, S_{3}, S_{4}, S_{5}\right) \quad \text { and } \quad \boldsymbol{V}=\left(V_{1}, V_{2}, V_{3}\right) .
$$

We now state the first main theorem.

THEOREM 1. There exists a polynomial $F(S ; V) \in R$ having the homogeneity of degrees with respect to $\boldsymbol{S}=\left(S_{0}, \ldots, S_{5}\right)$ such that

$$
F\left(T_{m} ; v_{m}\right)=0
$$

where $\boldsymbol{v}_{m}=\ell^{m}(v)$ and

$$
T_{m}=\left(\tau_{m-2}, \tau_{m-1}, \tau_{m}, \tau_{m+1}, \tau_{m+2}, \tau_{m+3}\right) .
$$

We can regard the sextet $(0.7)$ as the homogeneous coordinates of the complex projective space $\mathbb{C P}$ of dimension five. Then (0.6) defines a hypersurface in $\mathbb{C P}^{5}$, since the polynomial $F$ is homogeneous with respect to (0.7). The explicit form of $F$ will be given below in Section 1.2.

The Hamiltonian structure of the Painlevé system can be derived in a natural manner from the holonomic deformation of linear ordinary differential equations, see [4]. The algebraic relations of the $\tau$-functions of the second and third Painlevé systems have been studied in [1] by the use of the Schlesinger transformations of linear systems. On the other hand, in this paper we employ the method of the Hamiltonian structure for the fourth Painlevé system.

Consider, in addition to $\ell$, the translation

$$
\ell^{\prime}: v \longrightarrow v+e^{\prime}
$$

where $e^{\prime}=\frac{1}{3}(-1,2,-1)$. The two translations $\ell, \ell^{\prime}$ of the space $V=\{\boldsymbol{v}=$ $\left.\left(v_{1}, v_{2}, v_{3}\right) ; v_{1}+v_{2}+v_{3}=0\right\}$ generate the weight lattice group associated with 
the root system of the type $A_{2}$, see [3]. We can construct the birational canonical transformation

$$
\rho^{\prime}: \mathcal{H}(\boldsymbol{v}) \longrightarrow \mathcal{H}\left(\ell^{\prime}(\boldsymbol{v})\right)
$$

and then the algebraic variety

$$
F^{\prime}\left(T_{n}^{\prime} ; v_{n}^{\prime}\right)=0
$$

for the sequence $\left\{\tau_{n}^{\prime} ; n \in \mathbb{Z}\right\}$ of the $\tau$-functions. Here $\boldsymbol{v}_{n}^{\prime}=\left(\ell^{\prime}\right)^{n}(\boldsymbol{v})=\boldsymbol{v}+n \boldsymbol{e}^{\prime}$ and $T_{n}^{\prime}=\left(\tau_{n-2}^{\prime}, \tau_{n-1}^{\prime}, \tau_{n}^{\prime}, \tau_{n+1}^{\prime}, \tau_{n+2}^{\prime}, \tau_{n+3}^{\prime}\right)$. In Section 2.1 we will give the explicit form of the canonical transformation $\rho^{\prime}$ and establish the result analogous to Theorem 1 . Since the two translations $\ell$ and $\ell^{\prime}$ are commutative with each other, so are the transformations $\rho$ and $\rho^{\prime}$.

Let $\left\{\mathcal{H}_{(i, j)} ;(i, j) \in \mathbb{Z}^{2}\right\}$ be the family of the Painlevé systems such that, for an arbitrarily fixed value $\boldsymbol{v}$ of parameters,

$$
\begin{aligned}
& \mathcal{H}_{(0,0)}=\mathcal{H}(\boldsymbol{v}), \\
& \mathcal{H}_{(i, j)}=\rho^{i} \rho^{\prime j} \mathcal{H}_{(0,0)}=\mathcal{H}\left(\boldsymbol{v}_{(i, j)}\right),
\end{aligned}
$$

where $\boldsymbol{v}_{(i, j)}=v+i e+j e^{\prime}$. By putting $\mathcal{H}_{(i, j)}=\left(q_{(i, j)}, p_{(i, j)}, H_{(i, j)}, t\right)$, we obtain the family $\left\{\tau_{(i, j)} ;(i, j) \in \mathbb{Z}\right\}$ of $\tau$-functions which are defined by

$$
\frac{d}{d t} \log \tau_{(i, j)}=H_{(i, j)},
$$

with the ambiguity of a multiplicative constant. Let $R=\mathbb{Z}[S, V]$ be the polynomial ring considered above. The second main theorem is the following.

THEOREM 2. There exists a polynomial $F(S, V) \in R$, homogeneous with respect to $S$, such that for any $(i, j) \in \mathbb{Z}^{2}$,

$$
F\left(T_{(i, j)}, \boldsymbol{v}_{(i, j)}\right)=0
$$

where

$$
\boldsymbol{T}_{(i, j)}=\left(\tau_{(i-2, j-3)}, \tau_{(i-1, j-2)}, \tau_{(i, j-1)}, \tau_{(i+1, j)}, \tau_{(i+2, j+1)}, \tau_{(i+3, j+2)}\right) .
$$

Studies on the two-dimensional lattice of the $\tau$-functions related to the fourth Painlevé system will be shown in Section 3. 


\section{The sequence of $\tau$-functions}

\subsection{The birational canonical transformations}

In this subsection, we make a summary of explicit formulae concerning the birational canonical transformation:

$$
\rho: \mathcal{H}(\boldsymbol{v}) \longrightarrow \mathcal{H}(\ell(\boldsymbol{v})),
$$

of the fourth Painlevé system $\mathcal{H}=(q, p, H, t)$. Here $\ell$ is the translation

$$
\ell(v)=v+e \quad \text { with } e=\frac{1}{3}(-1,-1,2),
$$

of the space $V=\left\{\boldsymbol{v}=\left(v_{1}, v_{2}, v_{3}\right) ; v_{1}+v_{2}+v_{3}=0\right\} \subset \mathbb{C}^{3}$. As stated in the Introduction, we regard $V$ as the space of parameters of $\mathcal{H}$. For an arbitrary value $\boldsymbol{v} \in \boldsymbol{V}$ and for $m \in \mathbb{Z}$, we write

$$
\boldsymbol{v}_{m}=\ell^{m}(\boldsymbol{v})=\boldsymbol{v}+m e=\left(v_{1}-\frac{1}{3} m, v_{2}-\frac{1}{3} m, v_{3}+\frac{2}{3} m\right) .
$$

Then we obtain the sequence $\left\{\mathcal{H}_{m} ; m \in \mathbb{Z}\right\}$ of the Painlevé system:

$$
\begin{aligned}
& \mathcal{H}_{0}=\mathcal{H}(\boldsymbol{v}), \\
& \mathcal{H}_{m}=\rho^{m} \mathcal{H}_{0}=\mathcal{H}\left(\boldsymbol{v}_{m}\right)=\left(q_{m}, p_{m}, H_{m}, t\right),
\end{aligned}
$$

with the Hamiltonian

$$
H_{m}=2 q_{m} p_{m}^{2}-\left(q_{m}^{2}+2 t q_{m}+2\left(v_{2}-v_{1}\right)\right) p_{m}+\left(v_{3}-v_{1}+m\right) q_{m} .
$$

It follows that the Painlevé system of differential equations read

$$
\left\{\begin{array}{l}
\frac{d q_{m}}{d t}=\frac{\partial H_{m}}{\partial p_{m}}=4 q_{m} p_{m}-q_{m}^{2}-2 t q_{m}-2\left(v_{2}-v_{1}\right) \\
\frac{d p_{m}}{d t}=-\frac{\partial H_{m}}{\partial q_{m}}=-2 p_{m}^{2}+2\left(q_{m}+t\right) p_{m}-\left(v_{3}-v_{1}+m\right) .
\end{array}\right.
$$

Now we give the explicit form of the birational canonical transformation:

$$
\rho: \mathcal{H}_{m} \longrightarrow \mathcal{H}_{m+1} \text {. }
$$

The transformation consists of the contiguity relation

$$
\left\{\begin{array}{l}
q_{m}=-2 p_{m+1} \frac{X_{m+1}+v_{1}-v_{2}}{X_{m+1}+v_{1}-v_{3}-m-1} \\
p_{m}-\frac{1}{2} q_{m}-t=\frac{X_{m+1}+v_{1}-v_{3}-m-1}{2 p_{m+1}}
\end{array}\right.
$$


of the canonical variables, and the change of the Hamiltonian functions

$$
H_{m+1}=H_{m}+q_{m},
$$

where we set $X_{m}=q_{m} p_{m}$. It follows from (1.3) that

$$
\begin{gathered}
-X_{m}+\frac{1}{2} q_{m}^{2}+t q_{m}=X_{m+1}+v_{1}-v_{2}, \\
\left\{\begin{array}{l}
q_{m+1}=2\left(p_{m}-\frac{1}{2} q_{m}-t\right) \frac{X_{m+1}}{X_{m+1}+v_{1}-v_{3}-m-1}, \\
p_{m+1}=-\frac{1}{2} q_{m} \frac{X_{m+1}+v_{1}-v_{3}-m-1}{X_{m+1}+v_{1}-v_{2}} .
\end{array}\right.
\end{gathered}
$$

Since the system (1.2) implies the equation

$$
\frac{d X_{m+1}}{d t}=q_{m+1}\left(X_{m+1}+v_{1}-v_{3}-m-1\right)+2 p_{m+1}\left(X_{m+1}+v_{1}-v_{2}\right),
$$

we have from (1.3) the relation

$$
q_{m+1}-q_{m}=\frac{d}{d t} \log \left(X_{m+1}+v_{1}-v_{3}-m-1\right) .
$$

On the other hand, by the definition of the $\tau$-function, we deduce from (1.4) that

$$
q_{m+1}-q_{m}=\frac{d}{d t} \log \frac{\tau_{m+2} \tau_{m}}{\tau_{m+1}^{2}},
$$

which establishes the following proposition.

PROPOSITION 1.1. For any $m \in \mathbb{Z}$, there exists a nonzero constant $a(m)$ such that

$$
X_{m}+v_{1}-v_{3}-m=a(m) \frac{\tau_{m-1} \tau_{m+1}}{\tau_{m}^{2}} .
$$

By taking into consideration

$$
\frac{d H_{m}}{d t}=-2 q_{m} p_{m}=-2 X_{m},
$$

we arrive at the Toda equation for the $\tau$-functions:

$$
\frac{d^{2}}{d t^{2}} \log \tau_{m}+2\left(v_{3}-v_{1}+m\right)=-2 a(m) \frac{\tau_{m-1} \tau_{m+1}}{\tau_{m}^{2}} .
$$

If we replace $\tau_{m}$ in (1.7) by $f(m) \tau_{m}, f(m)$ being a nonzero constant, then $a(m)$ changes into

$$
a(m) \frac{f(m-1) f(m+1)}{f(m)^{2}} .
$$




\subsection{Proof of Theorem 1}

We now verify Theorem 1 and give an explicit form of the polynomial $F(S, V)$. First, we introduce new notation for the sake of simplicity of presentation. Put

$$
\begin{aligned}
B_{m} & =X_{m+1}+X_{m}+v_{1}-v_{2} \\
C_{m} & =X_{m} \frac{X_{m}+v_{1}-v_{2}}{X_{m}+v_{1}-v_{3}-m}
\end{aligned}
$$

Then it follows from (1.5) that

$$
\left\{\begin{array}{l}
\frac{1}{2} q_{m}^{2}+t q_{m}=B_{m}, \\
\frac{1}{2} q_{m-1}^{2}+t q_{m-1}=B_{m-1},
\end{array}\right.
$$

for $m \in \mathbb{Z}$ and, moreover, it follows from (1.3), (1.6) that

$$
q_{m} q_{m-1}=-2 C_{m} .
$$

We can deduce from (1.8) and (1.9) that

$$
q_{m}\left(B_{m-1}-C_{m}\right)=q_{m-1}\left(B_{m}-C_{m}\right)
$$

and then by again using (1.9) we have

$$
\left\{\begin{array}{l}
\frac{1}{2} q_{m}^{2}+\frac{B_{m}-C_{m}}{B_{m-1}-C_{m}} C_{m}=0 \\
\frac{1}{2} q_{m-1}^{2}+\frac{B_{m-1}-C_{m}}{B_{m}-C_{m}} C_{m}=0
\end{array}\right.
$$

Therefore we obtain the following.

PROPOSITION 1.2. For any $m \in \mathbb{Z}$,

$$
\frac{B_{m}-C_{m}}{B_{m-1}-C_{m}} C_{m}=\frac{B_{m}-C_{m+1}}{B_{m+1}-C_{m+1}} C_{m+1} .
$$

Theorem 1 is an immediate consequence of this proposition. In fact, equation (1.11) contains $X_{m-1}, X_{m}, X_{m+1}, X_{m+2}$, so that by virtue of (1.7) this defines an algebraic relation among the six $\tau$-functions: $\tau_{m-2}, \ldots, \tau_{m+3}$. It is not difficult to write (1.11) explicitly in terms of the $\tau$-functions; in fact we have 


$$
\begin{aligned}
a(m+ & 1)\left(a(m) \tau_{m-1} \tau_{m+1}+\left(v_{3}-v_{1}+m\right) \tau_{m}^{2}\right) \\
& \times\left(a(m) \tau_{m-1} \tau_{m+1}+\left(v_{3}-v_{2}+m\right) \tau_{m}^{2}\right) \\
& \times\left(a(m) a(m+1) \tau_{m-1} \tau_{m} \tau_{m+2}+a(m) \tau_{m-1} \tau_{m+1}^{2}\right. \\
& \left.-\left(v_{3}-v_{1}+m\right)\left(v_{3}-v_{2}+m\right) \tau_{m}^{2} \tau_{m+1}\right) \\
& \times\left(a(m+1) a(m)+2 \tau_{m} \tau_{m+1} \tau_{m+3}+a(m+1) \tau_{m} \tau_{m+2}^{2}\right. \\
& \left.-\left(v_{3}-v_{1}+m+1\right)\left(v_{3}-v_{2}+m+1\right) \tau_{m+1}^{2} \tau_{m+2}\right) \\
= & a(m)\left(a(m+1) \tau_{m} \tau_{m+2}+\left(v_{3}-v_{1}+m+1\right) \tau_{m+1}^{2}\right) \\
& \times\left(a(m+1) \tau_{m} \tau_{m+2}+\left(v_{3}-v_{2}+m+1\right) \tau_{m+1}^{2}\right) \\
& \times\left(a(m) a(m+1) \tau_{m-1} \tau_{m+1} \tau_{m+2}-a(m+1) \tau_{m}^{2} \tau_{m+2}\right. \\
& \left.-\left(v_{3}-v_{1}+m+1\right)\left(v_{3}-v_{2}+m+1\right) \tau_{m} \tau_{m+1}^{2}\right) \\
& \times\left(a(m-1) a(m) \tau_{m-2} \tau_{m} \tau_{m+1}-a(m) \tau_{m-1}^{2} \tau_{m}\right. \\
& \left.-\left(v_{3}-v_{1}+m\right)\left(v_{3}-v_{2}+m\right) \tau_{m-1} \tau_{m}^{2}\right) .
\end{aligned}
$$

By taking the normalization as $a(m-1)=a(m)=a(m+1)=-\frac{1}{2}$, we obtain the explicit form of $F(S, V)$ :

$$
\begin{aligned}
F(\boldsymbol{S}, \boldsymbol{V})= & \left(S_{1} S_{3}-2\left(V_{3}-V_{1}\right) S_{2}^{2}\right)\left(S_{1} S_{3}-2\left(V_{3}-V_{2}\right) S_{2}^{2}\right) \\
& \times\left(S_{1} S_{2} S_{4}-2 S_{1} S_{3}^{2}-4\left(V_{3}-V_{1}\right)\left(V_{3}-V_{2}\right) S_{2}^{2} S_{3}\right) \\
& \times\left(S_{2} S_{3} S_{5}-2 S_{2} S_{4}^{2}-4\left(V_{3}-V_{1}+1\right)\left(V_{3}-V_{2}+1\right) S_{3}^{2} S_{4}\right) \\
& -\left(S_{2} S_{4}-2\left(V_{3}-V_{1}+1\right) S_{3}^{2}\right)\left(S_{2} S_{4}-2\left(V_{3}-V_{2}+1\right) S_{3}^{2}\right) \\
& \times\left(S_{1} S_{3} S_{4}+2 S_{2}^{2} S_{4}-4\left(V_{3}-V_{1}+1\right)\left(V_{3}-V_{2}+1\right) S_{2} S_{3}^{2}\right) \\
& \times\left(S_{0} S_{2} S_{3}+2 S_{1}^{2} S_{2}-4\left(V_{3}-V_{1}\right)\left(V_{3}-V_{2}\right) S_{1} S_{2}^{2}\right)
\end{aligned}
$$

which is homogeneous of degree 10 with respect to $S=\left(S_{0}, \ldots, S_{5}\right)$.

\subsection{The Hamiltonian function $H_{m}$}

Viewing $\boldsymbol{T}_{m}=\left(\tau_{m-2}, \ldots, \tau_{m+3}\right)$ as a point of the projective space $\mathbb{C P}^{5}$, consider the algebraic variety $\mathfrak{V}_{m}$ defined by (1.11). In this section, we will investigate the function $t H_{m}$ on $\mathfrak{V}_{m}$. First note that the function $q_{m}^{2}$ is rational on $\mathfrak{V}_{m}$ by means of 
(1.10) and (1.8), and (1.11) implies the expression

$$
\left\{\begin{array}{l}
t q_{m}=B_{m}+\frac{B_{m}-C_{m}}{B_{m-1}-C_{m}} C_{m}, \\
t q_{m-1}=B_{m-1}+\frac{B_{m-1}-C_{m}}{B_{m}-C_{m}} C_{m} .
\end{array}\right.
$$

On the other hand, by multiplying these two equalities side by side and by using (1.9), we obtain

$$
q_{m}+q_{m-1}+2 t=\frac{1}{t}\left[C_{m}-\frac{B_{m} B_{m-1}}{C_{m}}\right] .
$$

We eliminate $q_{m}$ and $q_{m-1}$ from (1.12), (1.13) and then obtain the following proposition.

PROPOSITION 1.3.

$$
D_{m}+2 t^{2}=0 \quad \text { where } \quad D_{m}=\frac{\left(B_{m} B_{m-1}-C_{m}^{2}\right)^{2}}{\left(B_{m}-C_{m}\right)\left(B_{m-1}-C_{m}\right) C_{m}} .
$$

It is easy to verify that (1.11) implies the equality $D_{m}=D_{m+1}$. As a consequence, we can regard $t^{2}$ as a rational function on the variety $\mathfrak{V}_{m}$. Next we will verify the following proposition.

PROPOSITION 1.4. For any $m \in \mathbb{Z}, t H_{m}$ is rational on $\mathfrak{V}_{m}$.

In fact, by using (1.1), (1.3) we compute the Hamiltonian function $H_{m}$ as follows:

$$
H_{m}=2 p_{m}\left(X_{m}+v_{1}-v_{2}\right)-\left(q_{m}+2 t\right)\left(X_{m}+v_{3}-v_{1}+m\right),
$$

and then we obtain the expression

$$
\begin{aligned}
t H_{m} & =-t\left(X_{m}+v_{1}-v_{3}-m\right)\left(q_{m-1}+q_{m}+2 t\right) \\
& =-\left(X_{m}+v_{1}-v_{3}-m\right)\left(C_{m}-\frac{B_{m-1} B_{m}}{C_{m}}\right)-D_{m}\left(v_{1}-v_{3}-m\right) .
\end{aligned}
$$

The proposition is established by means of Proposition 1.3.

Put $U_{m}=-2 X_{m}-2\left(v_{1}-v_{3}-m\right)$. Then we obtain from the Toda equation and (1.7),

$$
U_{m}=-2 a(m) \frac{\tau_{m-1} \tau_{m+1}}{\tau_{m}^{2}}, \quad \frac{d U_{m}}{d t}=\left(q_{m}-q_{m-1}\right) U_{m}
$$


Here note that $t\left(q_{m}-q_{m-1}\right)$ is rational on $\mathfrak{V}_{m}$ by means of (1.12). Furthermore, we can verify the following differential equations:

$$
\begin{aligned}
\frac{d B_{m}}{d t} & =\left(q_{m}-q_{m-1}\right) U_{m}+\left(q_{m+1}-q_{m}\right) U_{m+1} \\
\frac{d C_{m}}{d t} & =-\frac{1}{2}\left(q_{m}-q_{m-1}\right)\left(U_{m}-\frac{4\left(v_{1}-v_{3}-m\right)\left(v_{2}-v_{3}-m\right)}{U_{m}}\right) .
\end{aligned}
$$

\section{The birational canonical transformation $\rho^{\prime}$}

In this section we consider the translation

$$
\ell^{\prime}: v \longrightarrow v+e^{\prime}, \quad e^{\prime}=\frac{1}{3}(-1,2,-1),
$$

of the space $V$ of parameters of the fourth Painlevé system. If we consider the reflection $s_{2}$ of $V$ defined by

$$
s_{2}:\left(v_{1}, v_{2}, v_{3}\right) \longrightarrow\left(v_{1}, v_{3}, v_{2}\right),
$$

then $\ell$ and $\ell^{\prime}$ are related to each other as follows:

$$
\ell^{\prime}=s_{2} \ell s_{2} \text {. }
$$

By using the same method as in [3], it is not difficult to construct the birational canonical transformation $\rho^{\prime}$, mapping $\mathcal{H}(\boldsymbol{v})$ to $\mathcal{H}\left(\ell^{\prime}(\boldsymbol{v})\right)$. We will give the explicit form of $\rho^{\prime}$ below.

\subsection{Other sequences of $\tau$-functions}

We define the sequence $\left\{\mathcal{H}^{(n)} ; n \in \mathbb{Z}\right\}$ of the Painlevé system by

$$
\begin{aligned}
& \mathcal{H}_{0}=\mathcal{H}(\boldsymbol{v}), \\
& \mathcal{H}^{(n)}=\left(\rho^{\prime}\right)^{n} \mathcal{H}_{0}=\mathcal{H}\left(\boldsymbol{v}^{(n)}\right)=\left(q^{(n)}, p^{(n)}, H^{(n)}, t\right),
\end{aligned}
$$

where $v^{(n)}=\left(\ell^{\prime}\right)^{(n)} v=v+n e^{\prime}$. The sequence $\left\{\tau^{(n)} ; n \in \mathbb{Z}\right\}$ of the $\tau$-functions is given by

$$
\frac{d}{d t} \log \tau^{(n)}=H^{(n)},
$$

up to multiplicative constants. By putting $X^{(n)}=q^{(n)} p^{(n)}$, we can write down $\rho^{\prime}$, the transformation from $\mathcal{H}^{(n)}$ to $\mathcal{H}^{(n+1)}$. In the remainder of this section, we do not enter into details of computation. 
PROPOSITION 2.1. For any $n \in \mathbb{Z}, \rho^{\prime}$ is composed of the contiguity relations

$$
\left\{\begin{array}{l}
q^{(n+1)}-2 p^{(n+1)}+2 t=2 \frac{X^{(n)}+v_{1}-v_{2}-n}{q^{(n)}} \\
p^{(n+1)}=-\frac{1}{2} q^{(n)} \frac{X^{(n)}+v_{1}-v_{3}}{X^{(n)}+v_{1}-v_{2}-n}
\end{array}\right.
$$

and the change of Hamiltonian functions

$$
H^{(n+1)}=H^{(n)}-2 p^{(n+1)} .
$$

Moreover, we have from (2.1) the relations

$$
\begin{gathered}
X^{(n)}+v_{1}-v_{3}=-X^{(n+1)}+2\left(p^{(n+1)}\right)^{2}-2 t p^{(n+1)}, \\
p^{(n)}=\frac{1}{2}\left(q^{(n+1)}-2 p^{(n+1)}+2 t\right) \frac{X^{(n)}}{X^{(n)}+v_{1}-v_{2}-n}, \\
q^{(n)}=-2 p^{(n+1)} \frac{X^{(n)}+v_{1}-v_{2}-n}{X^{(n)}+v_{1}-v_{3}} .
\end{gathered}
$$

By the definition of the $\tau$-functions, we obtain the expression

$$
2 p^{(n+1)}=\frac{d}{d t} \log \frac{\tau^{(n)}}{\tau^{(n+1)}},
$$

and then the following proposition.

PROPOSITION 2.2. There exists a nonzero constant $b(n)$ such that

$$
X^{(n)}+v_{1}-v_{2}-n=b(n) \frac{\tau^{(n-1)} \tau^{(n+1)}}{\left(\tau^{(n)}\right)^{2}} .
$$

\subsection{Relations for the $\tau$-functions}

The transformation $\rho^{\prime}$ is available to express

$$
B^{(n)}=X^{(n)}+X^{(n+1)}+v_{1}-v_{3}, \quad C^{(n)}=X^{(n)} \frac{X^{(n)}+v_{1}-v_{3}}{X^{(n)}+v_{1}-v_{2}-n} .
$$

Then we can verify the following propositions in a similar manner to proving (1.8), (1.9) and Proposition 1.2. 
PROPOSITION 2.3. For any $n \in \mathbb{Z}$, we have

(i)

(ii)

$$
\left\{\begin{array}{l}
B^{(n)}=2 p^{(n+1)}\left(p^{(n+1)}-t\right) \\
C^{(n)}=-2 p^{(n+1)} p^{(n)}
\end{array}\right.
$$

$$
\left\{\begin{array}{l}
2\left(p^{(n+1)}\right)^{2}+C^{(n)} \frac{B^{(n)}-C^{(n)}}{B^{(n-1)}-C^{(n)}}=0 \\
2\left(p^{(n)}\right)^{2}+C^{(n)} \frac{B^{(n-1)}-C^{(n)}}{B^{(n)}-C^{(n)}}=0
\end{array}\right.
$$

PROPOSITION 2.4.

$$
C^{(n)} \frac{B^{(n)}-C^{(n)}}{B^{(n-1)}-C^{(n)}}=C^{(n+1)} \frac{B^{(n)}-C^{(n+1)}}{B^{(n+1)}-C^{(n+1)}} .
$$

The equation given in Proposition 2.4 defines an algebraic relation among the six $\tau$-functions:

$$
\tau^{(n-2)}, \tau^{(n-1)}, \tau^{(n)}, \tau^{(n+1)}, \tau^{(n+2)}, \tau^{(n+3)} .
$$

We have thus established the analogous result of Theorem 1 with respect to the sequence $\left\{\tau^{(n)} ; n \in \mathbb{Z}\right\}$.

Moreover, we can obtain the following propositions, which correspond to Propositions 1.2, 1.3 and 1.4.

Proposition 2.5. For $n \in \mathbb{Z}$, we have

$$
\begin{gathered}
2 t p^{(n+1)}+\frac{B^{(n)} B^{(n-1)}-\left(C^{(n)}\right)^{2}}{B^{(n-1)}-C^{(n)}}=0, \\
2 t p^{(n)}+\frac{B^{(n)} B^{(n-1)}-\left(C^{(n)}\right)^{2}}{B^{(n)}-C^{(n)}}=0, \\
p^{(n+1)}+p^{(n)}-t=\frac{B^{(n)} B^{(n-1)}-\left(C^{(n)}\right)^{2}}{2 t C^{(n)}} .
\end{gathered}
$$

PROPOSITION 2.6. If we put

$$
D^{(n)}=\frac{\left(B^{(n)} B^{(n-1)}-\left(C^{(n)}\right)^{2}\right)^{2}}{C^{(n)}\left(B^{(n-1)}-C^{(n)}\right)\left(B^{(n)}-C^{(n)}\right)},
$$

then

$$
D^{(n)}+2 t^{2}=0
$$


Under the algebraic relation given in Proposition 2.4, we have $D^{(n+1)}=D^{(n)}$. PROPOSITION 2.7. For the Hamiltonian functions, we have

$$
\begin{gathered}
H^{(n)}-2 t\left(v_{1}-v_{2}-n\right)=2\left(p^{(n+1)}+p^{(n)}-t\right)\left(X^{(n)}+v_{1}-v_{2}-n\right), \\
t H^{(n)}=\left(X^{(n)}+v_{1}-v_{2}-n\right) \frac{B^{(n)} B^{(n-1)}-\left(C^{(n)}\right)-2}{C^{(n)}}-D^{(n)}\left(v_{1}-v_{2}-n\right) .
\end{gathered}
$$

Finally, put $U^{(n)}=-2 X^{(n)}-2\left(v_{1}-v_{2}-n\right)$. We can verify the relation

$$
U^{(n)}=-2 b(n) \frac{\tau^{(n-1)} \tau^{(n+1)}}{\left(\tau^{(n)}\right)^{2}},
$$

and then the differential equations

$$
\begin{aligned}
& \frac{d U^{(n)}}{d t}=-2\left(p^{(n+1)}-p^{(n)}\right) U^{(n)}, \\
& \frac{d B^{(n)}}{d t}=\left(p^{(n+1)}-p^{(n)}\right) U^{(n)}+\left(p^{(n+2)}-p^{(n+1)}\right) U^{(n+1)}, \\
& \frac{C^{(n)}}{d t}=\left(p^{(n+1)}-p^{(n)}\right)\left(U^{(n)}-\frac{4\left(v_{1}-v_{2}-n\right)\left(v_{3}-v_{2}-n\right)}{U^{(n)}}\right) .
\end{aligned}
$$

\section{Lattice of the $\tau$-functions}

In this section, we study the family $\left\{\mathcal{H}_{(i, j)} ;(i, j) \in \mathbb{Z}^{2}\right\}$ of the fourth Painlevé system and the family $\left\{\tau_{(i, j)} ;(i, j) \in \mathbb{Z}^{2}\right\}$ of the $\tau$-functions, where we put

$$
\begin{aligned}
& \mathcal{H}_{(0,0)}=\mathcal{H}(\boldsymbol{v}), \\
& \mathcal{H}_{(i, j)}=\rho^{i}\left(\rho^{\prime}\right)^{j} \mathcal{H}(\boldsymbol{v})=\mathcal{H}\left(\ell^{i}\left(\ell^{\prime}\right)^{j}(\boldsymbol{v})\right)=\left(q_{(i, j)}, p_{(i, j)}, H_{(i, j)}, t\right) .
\end{aligned}
$$

Note that $\rho$ and $\rho^{\prime}$ are commutative to each other. Because of the commutativity of the translation $\ell, \ell^{\prime}$, we have

$$
\ell^{i}\left(\ell^{\prime}\right)^{j}(v)=v+i e+j \boldsymbol{e}^{\prime}=\left(v_{1}-\frac{i+j}{3}, v_{2}-\frac{i-2 j}{3}, v_{3}+\frac{2 i-j}{3}\right) .
$$

\subsection{Birational canonical transformations}

First, we rewrite the formulae obtained in the preceding two sections. Since

$$
\begin{aligned}
& H_{(i+1, j)}=H_{(i, j)}+q_{(i, j)}, \\
& H_{(i, j)}=H_{(i, j+1)}+2 p_{(i, j+1)},
\end{aligned}
$$


by (1.4) and (2.2) respectively, we have

$$
\begin{aligned}
H_{(i+1, j+1)} & =H_{(i, j)}-2 p_{(i, j+1)}+q_{(i, j+1)} \\
& =H_{(i, j)}-2 p_{(i+1, j+1)}+q_{(i, j)} .
\end{aligned}
$$

We introduce the notation

$$
\begin{aligned}
& X_{(i, j)}=q_{(i, j)} p_{(i, j)}, \\
& Y_{(i, j)}=q_{(i, j)}\left(p_{(i, j)}-\frac{1}{2} q_{(i, j)}-t\right), \\
& Z_{(i, j)}=\left(q_{(i, j)}-2 p_{(i, j)}+2 t\right) p_{(i, j)} .
\end{aligned}
$$

Then it follows from (1.5), (2.3) and (3.1) that

$$
\left\{\begin{array}{l}
Z_{(i, j+1)}+X_{(i, j)}=v_{3}-v_{1}+i, \\
Y_{(i, j)}+X_{(i+1, j)}=v_{2}-v_{1}+j, \\
Z_{(i+1, j+1)}=Y_{(i, j)}+v_{3}-v_{2}+i+1-j .
\end{array}\right.
$$

Moreover, we deduce from (1.3) and (2.1) the following proposition.

PROPOSITION 3.1. The birational canonical transformation from $\mathcal{H}_{(i, j)}$ to $\mathcal{H}_{(i+1, j+1)}$ consists of contiguity relations of the form

$$
\begin{aligned}
q_{(i+1, j+1)}-2 p_{(i+1, j+1)}+2 t & =2 p_{(i+1, j)} \frac{X_{(i+1, j)}+v_{1}-v_{2}-j}{X_{(i+1, j)}} \\
& =-q_{(i, j)} \frac{Y_{(i, j)}+v_{3}-v_{2}+i+1-j}{Y_{(i, j)}+v_{1}-v_{2}-j}, \\
p_{(i+1, j+1)} & =-\frac{1}{2} q_{(i+1, j)} \frac{X_{(i+1, j)}+v_{1}-v_{3}-i-1}{X_{(i+1, j)}+v_{1}-v_{2}-j} \\
& =-\left(p_{(i, j)}-\frac{1}{2} q_{(i, j)}-t\right) \frac{Y_{(i, j)}+v_{1}-v_{2}-j}{Y_{(i, j)}},
\end{aligned}
$$

and the change (3.2) of the Hamiltonian functions.

By using these formulae, we verify the following proposition.

PROPOSITION 3.2. For any $(i, j) \in \mathbb{Z}^{2}$, there exists a nonzero constant $e(i, j)$ such that

$$
X_{(i, j)}=-\frac{1}{2} e(i, j) \frac{\tau_{(i+1, j+1)} \tau_{(i-1, j-1)}}{\tau_{(i, j)}^{2}} .
$$


In fact, by means of (3.2) we have

$$
\begin{aligned}
& H_{(i+1, j+1)}-H_{(i, j)}=q_{(i, j+1)}-2 p_{(i, j+1)}, \\
& H_{(i, j)}-H_{(i-1, j-1)}=q_{(i-1, j)}-2 p_{(i-1, j)},
\end{aligned}
$$

and then by the definition of the $\tau$-functions we have

$$
\frac{d}{d t} \log \frac{\tau_{(i+1, j+1)} \tau_{(i-1, j-1)}}{\tau_{(i, j)}^{2}}=q_{(i, j+1)}-2 p_{(i, j+1)}-q_{(i-1, j)}+2 p_{(i-1, j)} .
$$

On the other hand, we can deduce from Proposition 3.1 that the right-hand side of (3.4) is equal to

$$
\begin{aligned}
& \frac{-q_{(i-1, j)}\left(Y_{(i-1, j)}+v_{3}-v_{2}+i-j\right)+2 p_{(i-1, j)}\left(Y_{(i-1, j)}+v_{1}-v_{2}-j\right)}{Y_{(i-1, j)}+v_{1}-v_{2}-j} \\
& =\frac{d}{d t} \log \left(Y_{(i-1, j)}+v_{1}-v_{2}-j\right) .
\end{aligned}
$$

Here, to obtain the second equality, we have used the Hamiltonian system associated with $\mathcal{H}_{(i-1, j)}$. The proposition is then established by virtue of (3.3).

Since for any $(i, j) \in \mathbb{Z}^{2}$ we have

$$
\frac{d}{d t} H_{(i, j)}=-2 X_{(i, j)},
$$

the following theorem is deduced from Propositions 1.1, 2.2 and 3.2.

THEOREM 3. There exist families $\{c(i, j)\},\{d(i, j)\},\{e(i, j)\}$ of nonzero constants such that

$$
\begin{aligned}
\frac{d^{2}}{d t^{2}} \log \tau_{(i, j)} & =c(i, j) \frac{\tau_{(i-1, j)} \tau_{(i+1, j)}}{\tau_{(i, j)}^{2}}-2\left(v_{3}-v_{1}+i\right) \\
& =d(i, j) \frac{\tau_{(i, j-1)} \tau_{(i, j+1)}}{\tau_{(i, j)}^{2}}-2\left(v_{2}-v_{1}+j\right) \\
& =e(i, j) \frac{\tau_{(i-1, j-1)} \tau_{(i+1, j+1)}}{\tau_{(i, j)}^{2}} .
\end{aligned}
$$

COROLlaRY 3.1. For the lattice $\left\{\tau_{(i, j)}\right\}$ of the $\tau$-functions, we have

$$
\begin{aligned}
& e(i, j) \tau_{(i-1, j-1)} \tau_{(i+1, j+1)}+2\left(v_{3}-v_{1}+i\right) \tau_{(i, j)}^{2}-c(i, j) \tau_{(i-1, j)} \tau_{(i+1, j)}=0 \\
& e(i, j) \tau_{(i-1, j-1)} \tau_{(i+1, j+1)}+2\left(v_{2}-v_{1}+j\right) \tau_{(i, j)}^{2}-d(i, j) \tau_{(i, j-1)} \tau_{(i, j+1)}=0
\end{aligned}
$$




\subsection{Verification of Theorem 2}

We will verify Theorem 2 in this subsection. By the definition of $X_{(i, j)}, Y_{(i, j)}$ and $Z_{(i, j)}$, we have

$$
Y_{(i, j)}+Z_{(i, j)}=-2\left(p_{(i, j)}-\frac{1}{2} q_{(i, j)}-t\right)\left(p_{(i, j)}-\frac{1}{2} q_{(i, j)}\right) .
$$

On the other hand, we show the following proposition.

Proposition 3.3. For $(i, j) \in \mathbb{Z}^{2}$,

$$
2\left(p_{(i, j)}-\frac{1}{2} q_{(i, j)}-t\right)\left(p_{(i+1, j+1)}-\frac{1}{2} q_{(i+1, j+1)}-t\right)=-Y_{(i, j)} \frac{Z_{(i+1, j+1)}}{X_{(i+1, j)}} .
$$

In fact, by the use of (3.3), the first relation given in Proposition 3.1 can be written as

$$
2\left(p_{(i+1, j+1)}-\frac{1}{2} q_{(i+1, j+1)}-t\right)=-q_{(i, j)} \frac{Z_{(i+1, j+1)}}{X_{(i+1, j)}} .
$$

By multiplying both sides by $p_{(i, j)}-\frac{1}{2} q_{(i, j)}-t$ and then using the definition of $Y_{(i, j)}$, we obtain (3.7).

Now we introduce the notation

$$
B_{(i, j)}=Y_{(i, j)}+Z_{(i, j)}, \quad C_{(i, j)}=-\frac{Y_{(i-1, j)} Z_{(i, j+1)}}{X_{(i, j)}} .
$$

Then (3.6) and (3.7) are written as

$$
\begin{gathered}
B_{(i, j)}=-2\left(p_{(i, j)}-\frac{1}{2} q_{(i, j)}-t\right)\left(p_{(i, j)}-\frac{1}{2} q_{(i, j)}\right), \\
C_{(i+1, j)}=2\left(p_{(i+1, j+1)}-\frac{1}{2} q_{(i+1, j+1)}-t\right)\left(p_{(i, j)}-\frac{1}{2} q_{(i, j)}-t\right),
\end{gathered}
$$

respectively, from which we obtain the two relations

$$
\begin{gathered}
\frac{B_{(i, j)}-C_{(i+1, j)}}{p_{(i, j)}-\frac{1}{2} q_{(i, j)}-t}=-2 p_{(i, j)}+q_{(i, j)}-2 p_{(i+1, j+1)}+q_{(i+1, j+1)}+2 t, \\
\frac{B_{(i+1, j+1)}-C_{(i+1, j)}}{p_{(i+1, j+1)}-\frac{1}{2} q_{(i+1, j+1)}-t}=-2 p_{(i, j)}+q_{(i, j)}-2 p_{(i+1, j+1)}+q_{(i+1, j+1)}+2 t .
\end{gathered}
$$

It follows that, for $(i, j) \in \mathbb{Z}^{2}$,

$$
\begin{aligned}
& \left(p_{(i+1, j+1)}-\frac{1}{2} q_{(i+1, j+1)}-t\right)\left(B_{(i, j)}-C_{(i+1, j)}\right) \\
& \quad=\left(p_{(i, j)}-\frac{1}{2} q_{(i, j)}-t\right)\left(B_{(i+1, j+1)}-C_{(i+1, j)}\right) .
\end{aligned}
$$

Next, we show the following proposition. 
PROPOSITION 3.4. For $(i, j) \in \mathbb{Z}^{2}$, we have

$$
C_{(i+1, j)} \frac{B_{(i, j)}-C_{(i+1, j)}}{B_{(i+1, j+1)}-C_{(i+1, j)}}=C_{(i, j-1)} \frac{B_{(i, j)}-C_{(i, j+1)}}{B_{(i-1, j-1)}-C_{(i, j-1)}} .
$$

In fact, we deduce from (3.10) and (3.9) the relations

$$
\left\{\begin{array}{l}
2\left(p_{(i, j)}-\frac{1}{2} q_{(i, j)}-t\right)^{2}=C_{(i+1, j)} \frac{B_{(i, j)}-C_{(i+1, j)}}{B_{(i+1, j+1)}-C_{(i+1, j)}} \\
2\left(p_{(i+1, j+1)}-\frac{1}{2} q_{(i+1, j+1)}-t\right)^{2}=C_{(i+1, j)} \frac{B_{(i+1, j+1)}-C_{(i+1, j)}}{B_{(i, j)}-C_{(i+1, j)}} .
\end{array}\right.
$$

By replacing $(i+1, j+1)$ by $(i, j)$ in the latter, and then comparing this with the former, we obtain (3.11).

Note that, by the definition of $B_{(i, j)}$ and $C_{(i, j)}$, (3.11) concerns the eight quantities

$$
\begin{aligned}
& X_{(i, j-1)}, X_{(i+1, j)} ; \\
& Y_{(i-1, j-1)}, Y_{(i, j)}, Y_{(i+1, j+1)} ; \\
& Z_{(i-1, j-1)}, Z_{(i, j)}, Z_{(i+1, j+1)} .
\end{aligned}
$$

On the other hand, by the use of (3.3) and (1.1), we deduce from Theorem 3 that, for $(i, j) \in \mathbb{Z}^{2}$,

$$
\left\{\begin{array}{l}
X_{(i, j)}=-\frac{1}{2} e(i, j) \frac{\tau_{(i-1, j-1)} \tau_{(i+1, j+1)}}{\tau_{(i, j)}^{2}}, \\
Y_{(i-1, j)}=\frac{1}{2} d(i, j) \frac{\tau_{(i, j-1)} \tau_{(i, j+1)}}{\tau_{(i, j)}^{2}} \\
Z_{(i, j+1)}=\frac{1}{2} c(i, j) \frac{\tau_{(i-1, j)} \tau_{(i+1, j)}}{\tau_{(i, j)}^{2}} .
\end{array}\right.
$$

Moreover, by means of (3.3), (3.11) can be regarded as an algebraic relation among the six $\tau$-functions

$$
\tau_{(i-2, j-3)}, \tau_{(i-1, j-2)}, \tau_{(i, j-1)}, \tau_{(i+1, j)}, \tau_{(i+2, j+1)}, \tau_{(i+3, j+2)} .
$$

It is clear that this algebraic relation has the homogeneity of degrees with respect to these six $\tau$-functions. Hence we have established Theorem 2. 
By taking into consideration the ambiguity of multiplicative constants, we can normalize the $\tau$-functions as

$$
\tau_{(i, j)}\left(t_{0}\right)=1,
$$

$t_{0}$ being an arbitrarily fixed point of $\mathbb{C}$. If we replace $\tau_{(i, j)}$ by $f(i, j) \tau_{(i, j)}, f(i, j)$ being a nonzero constant, then the constants in (3.13) are converted into the form

$$
\begin{gathered}
c(i, j) \cdot \frac{f(i-1, j) f(i+1, j)}{f(i, j)^{2}}, \quad d(i, j) \cdot \frac{f(i, j-1) f(i, j+1)}{f(i, j)^{2}}, \\
e(i, j) \cdot \frac{f(i-1, j-1) f(i+1, j+1)}{f(i, j)^{2}}
\end{gathered}
$$

respectively. By choosing suitable $f(i, j)$, we can assume one of the constants to be one for any $(i, j) \in \mathbb{Z}^{2}$.

We can regard (3.11) as an algebraic relation among the twelve $\tau$-functions

$$
\begin{aligned}
& \tau_{(i+2, j+2)}, \\
& \tau_{(i+1, j+1)}, \quad \tau_{(i+2, j+1)}, \\
& \tau_{(i, j)}, \quad \tau_{(i+1, j)}, \quad \tau_{(i+2, j)}, \\
& \tau_{(i-1, j-1)}, \quad \tau_{(i, j-1)}, \quad \tau_{(i+1, j-1)}, \\
& \tau_{(i-2, j-2)}, \quad \tau_{(i-1, j-2)}, \quad \tau_{(i, j-2)} .
\end{aligned}
$$

This relation is reduced to that concerning the sextet (3.14), by virtue of the quadratic relations stated above in Corollary 3.1 to Theorem 3 .

\subsection{The Hamiltonian function $H_{(i, j)}$}

We will give some expressions of the canonical variables of the Painlevé system $\mathcal{H}_{(i, j)}$ in terms of the $\tau$-functions. We obtain from (3.8) and (3.12)

$$
\begin{gathered}
2 t\left(p_{(i, j)}-\frac{1}{2} q_{(i, j)}-t\right)=-\frac{B_{(i, j)} B_{(i+1, j+1)}-C_{(i+1, j)}^{2}}{B_{(i+1, j+1)}-C_{(i+1, j)}}, \\
2 t\left(p_{(i+1, j+1)}-\frac{1}{2} q_{(i+1, j+1)}-t\right)=-\frac{B_{(i, j)} B_{(i+1, j+1)}-C_{(i+1, j)}^{2}}{B_{(i, j)}-C_{(i+1, j)}} .
\end{gathered}
$$

By substituting these equalities into (3.9), we have

$$
D_{(i+1, j)}=2 t^{2}
$$


where

$$
D_{(i, j)}=\frac{\left(B_{(i-1, j)} B_{(i, j+1)}-C_{(i, j)}^{2}\right)^{2}}{C_{(i, j)}\left(B_{(i-1, j)}-C_{(i, j)}\right)\left(B_{(i, j+1)}-C_{(i, j)}\right)} .
$$

It is not difficult to show that, under the algebraic relation (3.11),

$$
D_{(i+1, j)}=D_{(i, j-1)} .
$$

Moreover, we verify the following proposition.

PROPOSITION 3.5. The Hamiltonian function $H_{(i, j)}$ of $\mathcal{H}_{(i, j)}$ can be written as

$$
\begin{gathered}
H_{(i, j)}+2 t\left(v_{2}-v_{1}+j\right)=-2 Y_{(i-1, j)}\left(p_{(i, j)}+p_{(i, j+1)}-t\right), \\
H_{(i, j)}+2 t\left(v_{3}-v_{1}+i\right)=Z_{(i, j+1)}\left(q_{(i-1, j)}+q_{(i, j)}+2 t t\right), \\
H_{(i, j)}=-2 X_{(i, j)}\left(p_{(i, j+1)}-\frac{1}{2} q_{(i, j+1)}+p_{(i-1, j)}-\frac{1}{2} q_{(i-1, j)}-t\right) .
\end{gathered}
$$

In fact, the first one is nothing but the relation given in Proposition 2.5, while the second one is (1.14) of Section 1.3. Moreover, we obtain from (1.14) the equalities

$$
\begin{aligned}
H_{(i, j)} & =2 p_{(i, j)}\left(X_{(i, j)}-v_{2}+v_{1}-j\right)-q_{(i, j)}\left(X_{(i, j)}-v_{3}+v_{1}-i\right)+2 t X_{(i, j)} \\
& =-2 p_{(i, j)} Y_{(i-1, j)}+q_{(i, j)} Z_{(i, j+1)}+2 t X_{(i, j)} .
\end{aligned}
$$

On the other hand, we can deduce from the relations given in Proposition 3.1 that

$$
\begin{aligned}
& 2 p_{(i, j)} Y_{(i-1, j)}=2\left(p_{(i, j+1)}-\frac{1}{2} q_{(i, j+1)}-t\right), \\
& q_{(i, j)} Z_{(i, j+1)}=-2\left(p_{(i-1, j)}-\frac{1}{2} q_{(i-1, j)}-t\right) .
\end{aligned}
$$

The third relation of Proposition 3.5 is an immediate consequence of these equalities.

Finally, we construct the table of contiguity relations used above:

$$
\begin{aligned}
& \left\{\begin{array}{l}
q_{(i+1, j+1)}-2 p_{(i+1, j+1)}+2 y=q_{(i, j)} \frac{Z_{(i+1, j+1)}}{X_{(i+1, j)}}, \\
p_{(i+1, j+1)}=\left(p_{(i, j)}-\frac{1}{2} q_{(i, j)}-t\right) \frac{X_{(i+1, j)}}{Y_{(i, j)}},
\end{array}\right. \\
& \left\{\begin{array}{l}
q_{(i+1, j)}=-2\left(p_{(i, j)}-\frac{1}{2} q_{(i, j)}-t\right) \frac{X_{(i+1, j)}}{Z_{(i+1, j+1)}} \\
p_{(i+1, j)}=-\frac{1}{2} q_{(i, j)} \frac{Z_{(i+1, j+1)}}{Y_{(i, j)}},
\end{array}\right.
\end{aligned}
$$




$$
\left\{\begin{array}{l}
q_{(i, j+1)}-2 p_{(i, j+1)}+2 t=-2 p_{(i, j)} \frac{Y_{(i-1, j)}}{X_{(i, j)}}, \\
p_{(i, j+1)}=-\frac{1}{2} q_{(i, j)} \frac{Z_{(i, j+1)}}{Y_{(i-1, j)}} .
\end{array}\right.
$$

\section{REFERENCES}

[1] M. Jimbo and T. Miwa. Monodromy preserving deformation of linear ordinary differential equations with rational coefficients II. Physica 2D (1981), 407-448.

[2] K. Okamoto. On the $\tau$-function of the Painlevé equations. Physica 2D (1981), 525-535.

[3] K. Okamoto. Studies on the Painlevé equations III: second and fourth Painlevé equations, $P_{I I}$ and $P_{I V}$. Math. Ann. 275 (1986), 221-255.

[4] K. Okamoto. Isomonodromic deformation and the Painlevé equations, and the Garnier system. J. Fac. Sci. Univ. Tokyo Sect. IA Math. 33 (1986), 575-618.

\section{Kazuo Okamoto}

Department of Mathematical Sciences

University of Tokyo

Komaba, Meguro, Tokyo, 153 Japan 